\title{
A 32-year-old woman with breast swelling and crepitant rales
}

\begin{abstract}
32-year-old previously A healthy female smoker was admitted to hospital after presenting with a sudden onset of painful bilateral breast swelling and chest pain that occurred when she was resting. Her pain was nonpleuritic, with no associated shortness of breath, diaphoresis, nausea or radiation. On palpation, her breasts and chest $\infty$ wall were swollen, and on aus-
$\exists$ cultation, crepitant rales were heard. The rest of her examination was normal, as were her blood chemistry, cardiac enzyme levels, leukocyte count, hematocrit and electrocardiogram. A chest radiograph revealed large
\end{abstract}
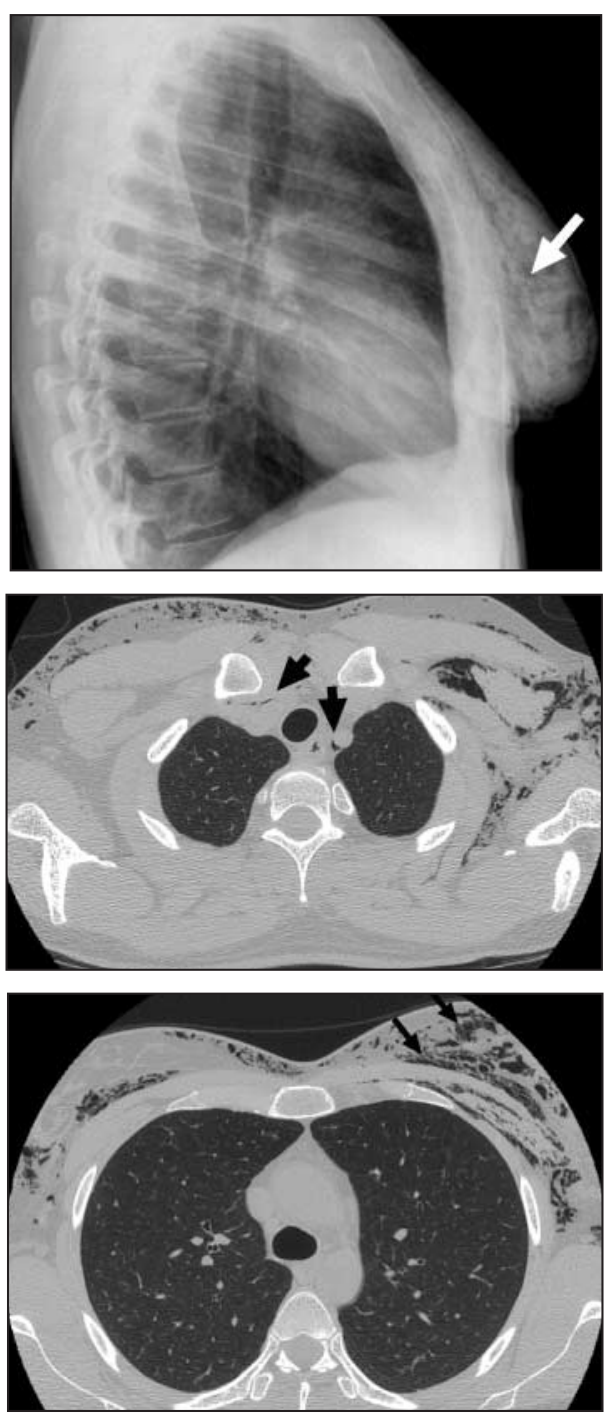

amounts of gas within the mammary parenchyma (Fig. 1, arrow), without evidence of pneumothorax. A CT scan showed pneumomediastinum (Fig. 2, arrows) and extensive subcutaneous and mammary emphysema (Fig. 3, arrows), but no underlying lung disease. A barium test did not reveal any esophageal leakage. Secondary causes of pneumomediastinum, including asthma, cocaine consumption, trauma or recent invasive procedures, were excluded. The patient's pain was successfully controlled with acetaminophen, and she did not need oxygen supplementation. Subsequent chest radiographs showed spontaneous recovery of the air collections over about a week, and the patient was discharged.

Pneumomediastinum, defined as the presence of air in the mediastinum, may result from several causes, including chest trauma, airway or esophageal rupture, endobronchial or esophageal procedures, mechanical ventilation or chest surgery. Air often spreads through tissue planes, leading to subcutaneous emphysema with or without pneumoperitoneum, pneumoretroperitoneum, pneumopericardium or pneumothorax. Spontaneous pneumomediastinum may occur without obvious cause, and it is believed to result from the rupture of marginally situated alveoli caused by a rapid increase in alveolar pressure, followed by centripetal gas dissection through the pulmonary interstitium into the mediastinum. ${ }^{1}$ Predisposing factors include inhalational drug abuse, labour, extreme coughing or other activities associated with Valsalva's manoeuvre. ${ }^{2}$ Pneumomediastinum itself is usually benign and self-limiting, with complications or attributable to the precipitating condition; aggressive air leak drainage or surgical intervention have been used in cases of cardiorespiratory compromise.

Mammary emphysema is an uncommon manifestation of pneumomediastinum. Gas does not usually penetrate the breast because the superficial mammary fascia separates the glandular tissue from the retromammary space, which is in turn separated from the pectoralis major muscle by the deep pectoral fascia. Two reported cases of mammary emphysema were both complications of hospital procedures (mechanical ventilation and insertion of a chest tube). ${ }^{3,4}$ In our patient, gas may have dissected anteriorly into her breast along 1 of 2 routes: through the superolateral portion (the axillary tail of Spence), which is the only area of breast tissue that penetrates the superficial fascia into the deep pectoral fascia, or through the small projections of mammary tissue that may occasionally penetrate the deep pectoral fascia into the pectoralis major. ${ }^{5}$ Breast swelling and crepitant rales should be considered important clinical signs of possible underlying pneumomediastinum.

\section{Elmo Mannarino \\ Graziana Lupattelli \\ Giuseppe Schillaci \\ Unit of Internal Medicine \\ Angiology and Arteriosclerosis \\ University of Perugia \\ Perugia, Italy}

\section{References}

1. Gerazounis M, Athanassiadi K, Kalantzi N, Moustardas M. Spontaneous pneumomediastinum: a rare benign entity. $\mathcal{F}$ Thorac Cardiovasc Surg 2003;126:774-6.

2. Panacek EA, Singer AJ, Sherman BW, Prescott A, Rutherford WF. Spontaneous pneumomediastinum: clinical and natural history. Ann Emerg Med 1992;21:1222-7.

3. Vargas FC, Vas W, Carlin B, Morris L, Salimi Z. Radiographic and CT demonstration of mammary emphysema. $\mathcal{F}$ Comput Assist Tomogr 1985;9:560-2.

4. Gold BM, Perlmutter S. Emphysema of the breast simulating fibroadenolipoma. Am 7 Roentgenol 1994;162:730.

5. Warwick R, William PL, editors Gray's anatomy. 35th ed. Philadelphia: WB Saunders; 1973. p. 1364-7. 